\title{
Red-giant branch morphology of metal-poor globular clusters in the Galactic bulge
}

\author{
J. W. Kim ${ }^{1}$, H. I. Kim ${ }^{2}$ and Y. J. Sohn ${ }^{1}$ \\ ${ }^{1}$ Department of Astronomy, IEAA, Yonsei University, Seoul 120-749, Korea \\ email: kjw0704@galaxy.yonsei.ac.kr \\ ${ }^{2}$ Korea Astronomy and Space Science Institute, Daejeon 305-348, Korea
}

\begin{abstract}
Using the $(J-K, K)$ color-magnitude diagrams (CMDs) of 16 metal-poor globular clusters in the Galactic bulge, we investigate the morphological properties of their red-giant branch (RGB), comparing with those of metal-rich clusters in the Galactic bulge and metalpoor clusters in the Galactic halo. The RGB morphological parameters, such as colors at fixed magnitudes, magnitudes at a fixed color, the RGB slope, and a difference of color indices at two fixed magnitudes have been derived from the near-IR CMDs for each cluster. Metal-poor Galactic bulge clusters follow the previous empirical relations between colors at fixed magnitudes and magnitudes at a fixed color of the RGB and the cluster's metallicity. However, the RGB slope and the color difference parameters of some bulge clusters deviate slightly from the previous empirical linear relations for the other globular clusters, implying that the metal-poor bulge clusters may have different formation origin from the other globular clusters in the Galaxy.
\end{abstract}

Keywords. Galaxy: structure - Galaxy: globular cluster: general — infrared: stars

\section{Introduction}

The Galactic bulge as a whole is a high metallicity domain where most stars have nearsolar metallicity, while bulk of stellar populations in the bulge is old without significant amount of recent star formation (e.g. Zoccali et al. 2003). In this respect, metal-poor globular clusters in the bulge are rather unique objects embedded in the metal-rich environment. Among a total of 20 metal-poor bulge globular clusters located within $3 \mathrm{kpc}$ of the Galactic center with metallicities $[\mathrm{Fe} / \mathrm{H}]<-1.0$ (Harris 1996), $J$ and $K$ images of 16 clusters are used to derive the RGB morphological parameters in their nearIR CMDs. Near-IR photometry offers advantages in studying the RGB population of the bulge globular clusters because of its relatively low extinction and high sensitivity to low temperature. By comparing the RGB morphology of metal-rich bulge clusters and metalpoor halo clusters (e.g. Kim et al. 2006), one can constrain theories of the formation and early evolution of the Galactic bulge.

\section{The RGB morphology}

The images of target clusters were obtained during two runs in 2003 and 2004 using the CFHTIR infrared imager of the $3.6 \mathrm{~m}$ CFHT. On the obtained $(J-K, K)$ CMDs of the resolved stars in the clusters, we define the fiducial normal points of the RGB with a typical bin of 0.25 magnitude in $K$ as Kim et al. (2006), from which we derive $(J-K)$ colors at fixed magnitudes $M_{K}=(-5.5,-5,-4$ and -3$)$, and $M_{K}$ magnitudes at a fixed color $(J-K)=0.7$. Figure 1 shows the color and magnitude parameters as a function of cluster's metallicity for the metal-poor bulge globular clusters, and they follow the 

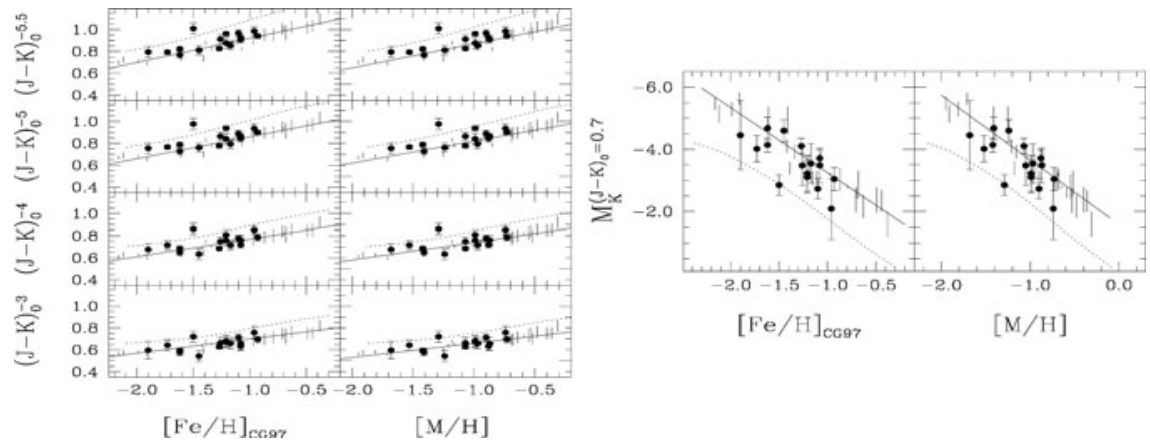

Figure 1. The $(J-K)$ colors at fixed magnitudes (left), and the $M_{K}$ magnitudes at a fixed color (right) as functions of cluster's metallicities $[\mathrm{Fe} / \mathrm{H}]_{C G 97}$ and $[\mathrm{M} / \mathrm{H}]$. Vertical bars indicate the previously determined parameters for metal-poor halo clusters and metal-rich bulge clusters, and the solid lines are the relationship by Valenti et al. $(2004,2005)$. Dotted line represents the prediction of Yonsei-Yale isochrones (Yi et al. 2003) at 12 Gyr.
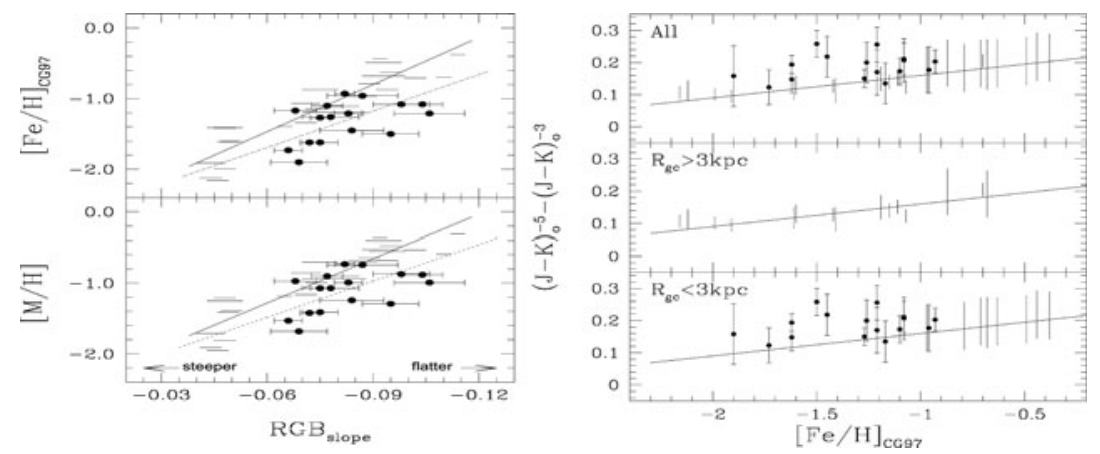

Figure 2. The RGB slope (left) and the color difference (right) as functions of cluster's metallicity. The dotted lines of left panel are the relations found by Ivanov \& Borissova (2002). The other symbols are the same as Fig. 1.

previously determined empirical relations for metal-poor halo clusters and metal-rich bulge clusters (Valenti et al. 2004, 2005).

RGB slopes are determined by fitting a linear equation to the bright upper part of the $\mathrm{RGB}$, and the difference of $(J-K)$ colors at $M_{K}=-3$ and -5 are also estimated on the fiducial normal points of the RGB. In Figure 2, RGB slopes (left) and color differences (right) for some metal-poor bulge globular clusters as a function of the cluster's metallicity show slightly different trends from the previous empirical relations, compared to the other metal-poor halo clusters and metal-rich bulge clusters (cf. Valenti et al. 2004, 2005). This may be a hint for a different formation origin of the metal-poor bulge globular clusters.

\section{References}

Harris, W. E. 1996, AJ 112, 1487

Ivanov, V. D. \& Borissova, J. 2002, A\&A 390,937

Kim, J.-W. et al. 2006, A\&A 459, 499

Valenti, E., Ferraro, F. R., \& Origlia, L. 2004, MNRAS 351, 1204

Valenti, E., Origlia, L. \& Ferraro, F. R. 2005, MNRAS 361, 272

Yi, S. K., Kim, Y. -C., \& Demarque, P. 2003, ApJS 144, 259

Zoccali, M. et al. 2003, A\&A 399, 931 